\title{
Les catholiques en Irlande du nord face a l'Europe
}

\author{
Adrian Guelke
}

\section{(2) OpenEdition}

\section{Journals}

\section{Édition électronique}

URL : http://journals.openedition.org/conflits/662

DOI : $10.4000 /$ conflits.662

ISSN : 1777-5345

Éditeur :

CCLS - Centre d'études sur les conflits lilberté et sécurité, L'Harmattan

\section{Édition imprimée}

Date de publication : 22 octobre 1992

ISSN : 1157-996X

\section{Référence électronique}

Adrian Guelke, «Les catholiques en Irlande du nord face a l'Europe », Cultures \& Conflits [En ligne], 07 | automne 1992, mis en ligne le 06 janvier 2003, consulté le 30 mars 2021. URL : http:// journals.openedition.org/conflits/662 ; DOI : https://doi.org/10.4000/conflits.662

Ce document a été généré automatiquement le 30 mars 2021.

Creative Commons License 


\title{
Les catholiques en Irlande du nord face a l'Europe
}

\author{
Adrian Guelke
}

Les profondes divisions sectaires qui caractérisent l'Irlande du Nord sont simultanément cause et effet du conflit qui la déchirent. Néanmoins, l'antagonisme entre protestants et catholiques ne s'explique pas uniquement en termes de dynamique interne de la société nord-irlandaise; il ne renvoie pas non plus à une guerre de religion : ce qui est en jeu est la perception même de l'identité nationale. L'Irlande du Nord doit-elle, comme le souhaitent les protestants, continuer de faire partie du Royaume-Uni ou doit-elle faire partie d'une Irlande unie, comme le voudraient les catholiques? L'intégration de l'Irlande du Nord au Royaume-Uni est "conditionnelle", elle repose sur la volonté de la majorité de la population de la province, aussi cette province n'a-t-elle pas été pleinement intégrée dans le système politique intérieur britannique: examinée d'un point de vue international, la situation dé l'Irlande du Nord n'est donc pas immuable, ce qui, on s'en doute, ne manque pas de contribuer au conflit. Malgré une coopération certaine entre la Grande-Bretagne et la République d'Irlande, la violence du conflit n'est pas entièrement contrôlée, le statut de la province n'est pas clarifié et la minorité catholique n'est pas encore réconciliée avec l'existence de l'Irlande du Nord en tant qu'unité politique. Le nouveau contexte que constitue à la fois la désintégration de l'Union soviétique et celle de la Yougoslavie - ce qui a ouvert des perspectives de changements de frontières en Europe - et les progrès enregistrés dans la création d'une Union européenne, font miroiter l'espoir qu'une solution au statut de l'Irlande du Nord pourrait être facilitée par ce nouveau cadre politique. Nous nous proposons d'étudier dans cet article la perception qu'ont les catholiques de ces transformations.

L'attitude des partis nationalistes face à l'Europe de Maastricht

Les catholiques constituent $40 \%$ da la population totale d'une province qui compte 1,5 millions d'habitants. Leur représentation dans l'électorat est légèrement inférieure à ce pourcentage. Ils adhèrent à trois partis politiques. La majorité des électeurs catholiques soutient le "parti social-démocrate et du travail" (SDLP), parti généralement considéré comme la voix d'un nationalisme constitutionnel, car il défend l'idée d'une Irlande unie 
dans le cadre d'un consensus démocratique. Entre un quart et un tiers de l'électorat catholique appuie un autre parti nationaliste irlandais, le Sinn Fein, aile politique de l'armée provisoire irlandaise" (IRA), tandis qu'environ $10 \%$ se rallie à l'Alliance Party, modéré et non sectaire, qui -contrairement aux deux autres partis - soutient l'union avec la Grande-Bretagne. Très peu de catholiques votent pour l'Ulster Unionist Party (I1UP) ou le Democratic Unionist Party (DUP), les deux partis "unionistes" qui regroupent une large majorité du vote protestant. Le SDLP soutient tout comme l'Alliance Party, l'intégration européenne, en revanche le Sinn Fein est hostile à la Communauté européenne. Le parti le plus fermement européen est le SDLP qui entrevoit une solution au problème de l'Irlande du Nord grâce à la Communauté européenne. Cependant, cet enthousiasme pour une "Europe des régions" n'est partagé par aucun autre parti de l'Irlande du Nord. Ceci est ressorti clairement des discussions inter-partis de 1992, où le SDLP a présenté un plan pour le gouvernement de l'Irlande du Nord calqué sur le fonctionnement de la Commission européenne.

A première vue, il paraît difficile pour un parti nationaliste irlandais d'accepter l'intégration européenne compte tenu de l'accent que mettent traditionnellement les nationalistes irlandais sur l'idéal d'une Irlande pleinement souveraine et indépendante. Aussi, considérée d'un point de vue historique, l'attitude actuelle du SDLP et des autres partis nationalistes irlandais d'inspiration constitutionnaliste à l'égard de l'intégration européenne représente un changement de position significatif. Cette constatation a été clairement exprimée par Brian Walker dans une déclaration faite à l'Irish Times après que le référendum de juin 1992, organisé en république d'Irlande sur le traité de Maastricht, ait donné une majorité de $70 \%$ des votes exprimés en faveur du traité : "Le résultat du vote sur le traité de Maastricht montre que les positions politiques traditionnelles, tant au nord au sud, ont subi un revirement d'importance historique. La majorité des nationalistes irlandais a manifesté sans équivoque son soutien à l'union renforcée entre les 12 membres de la Communauté européenne, tandis que les unionistes de l'Irlande du Nord, par contre, des nationalistes européens qui s'opposent à toute Union européenne renforcée"1.

Walker illustrait le résultat du référendum dans le Sud par le fait qu'au Nord huit des neuf députés à Westminster de I'Ulster Unionist Party (UUP) avaient soutenu une motion s'opposant au traité de Maastricht à la Chambre des communes, alors que les quatre députés du SDLP soutenaient eux, le traité.

Néanmoins, tous les catholiques ne sont pas pro-européens. Pour le Sinn Fein, l'intégration européenne est un obstacle à la réalisation d'une Irlande souveraine et indépendante, appliquant la politique de neutralité, qui demeure son objectif traditionnel. Ce Parti existant au Sud, a participé au référendum sur la ratification du traité de Maastricht. Sous la manchette "Votez NON le 18 juin", son hebdomadaire, An Phoblacht, encourageait ses sympathisants du Sud à refuser le traité. L'argumentation utilisée était la suivante: "Un vote négatif arrêtera la course effrénée vers un super État européen centralisé où les parlements nationaux n'auront que peu de pouvoir et où la politique sera décidée par une bureaucratie non élue et par des commissions politiques dans lesquelles les 26 comtés ( $1 \%$ de la population de la Communauté européenne) n'auront qu'une voix minuscule et aucun droit de veto. Maastricht sonne le glas de la neutralité et d'une politique étrangère indépendante. Le traité engage le gouvernement de Dublin dans la politique étrangère et de défense de l'Union de l'Europe Occidentale, qui est le pilier européen de l'OTAN"2. Puis l'article présentait les raisons d'ordre social et économique qui militaient en faveur d'un vote négatif, et 
finalement abordait les implications de l'intégration européenne sur la scission de l'Irlande : "II n'y a rien dans ce traité qui permette d'aborder le problème de la division politique et économique de l'Irlande. Tandis que les frontières européennes sont censées disparaître, la frontière ici continue à être renforcée et le degré de souveraineté du peuple irlandais diminue"3. II faut pourtant rappeler que même au sein de la minorité de l'électorat sudiste qui votait "non", les arguments apportés par le Sinn Fein n'ont eu que peu d'impact; la campagne du "non" étant dominée par les thèses contre l'avortement.

L'Europe : un vieil espoir

Alors que la plupart des nationalistes constitutionnalistes se sont détournés des thèses sur l'indépendance développées par le Sinn Fein, l'idée que la dimension européenne pourrait apporter de l'eau au moulin des nationalistes de l'Irlande du Nord a mûri lentement. C'est le ministre des Affaires étrangères de la république d'Irlande, Liam Cosgrave, qui le premier, en 1954, avait évoqué l'idée que l'Europe pourrait constituer un contexte pour dépasser les divisions irlandaises. D'une manière beaucoup plus générale, la classe politique de l'Irlande du Sud a beaucoup plus considéré l'Europe comme un facteur de réduction de la dépendance de l'Irlande par rapport à la GrandeBretagne que comme une solution à la division du pays. Cette focalisation sur les rapports entre l'Irlande et la Grande-Bretagne était particulièrement vive parmi les hommes politiques qui, comme Cosgrave, adhéraient au Fine Gael, le moins nationaliste des deux grands partis de l'Irlande. Pendant les années 60 et les premières années de 70 , alors que la probabilité d'une participation de la Grande-Bretagne et de -l'Irlande à la Communauté européenne croissait, les unionistes libéraux de l'Irlande du Nord étaient parmi ceux qui s'intéressaient aux conséquences que cet événement pourrait produire sur les relations entre les deux parties de l'Irlande. Ils espéraient que l'adhésion à la Communauté européenne provoquerait une modernisation accélérée de l'économie nord-irlandaise. La frontière perdant ainsi son rôle de barrière économique entre Nord et Sud, cette situation entraînerait par voie de conséquence un affaiblissement des divisions sectaires. Les horizons plus vastes de l'Europe devaient réduire le rôle négatif que joue la frontière dans le monde politique de la province.

Le point de vue des unionistes libéraux était clairement exprimé par le ministre du Commerce de l'Irlande du Nord, lors de la discussion sur l'adhésion à la Communauté européenne du Parlement de l'Irlande du Nord: "L'idéologie porteuse de l'Europe, ditil, n'a pas de place pour les haines et amertumes d'hier. Il va falloir décider si nous souhaitons nous enfermer dans ce cul-de-sac de l'histoire, ou si nous voulons rejoindre l'Europe du progrès. Je n'ai aucune sympathie particulière pour l'idéologie du Sinn Fein ou de ses équivalents qui exprime la plupart du temps une vue étroite de l'Ulster" ${ }^{\prime \prime}$.

Parmi les nationalistes de la république du Sud, Garret FitzGerald, s'exprimant en faveur d'une Irlande fédérée, considérait que la dimension européenne aurait un effet sur la division de l'île, l'adhésion à la Communauté jouant "exclusivement en faveur d'une Irlande unifiée"s. Dans son livre Towards a New Ireland (Vers une nouvelle Irlande), il présente six arguments en faveur de sa conclusion. Premièrement, il considère que le contact plus fréquent avec les autres Européens fera apparaître comme moins significatives les différences internes de l'Irlande. Deuxièmement, il prévoit que les intérêts communs en matière d'agriculture et de politique régionale dans le cadre de la Communauté des deux Irlandes, les rapprocheront effectivement. Troisièmement, avec l'adhésion à la Communauté disparaitrait toute justification économique de droits de douane protectionnistes. Quatrièmement, les disparités en 
matière de protection sociale entre le Sud et le Nord auront tendance à s'estomper. Cinquièmement, l'impact émotionnel d'un transfert de pouvoir de Westminster à Dublin serait amenuisé dans la mesure où une partie significative de ces prérogatives aurait déjà été transférée à Bruxelles. Finalement, il considère que la structure des institutions européennes favorisera considérablement les petits États et que, de ce fait, le Nord pourrait prendre conscience que s'il faisait partie d'une Irlande unie, il étofferait sa représentation au sein des institutions communautaires ${ }^{6}$.

Malgré les différences politiques profondes qui séparent un nationaliste constitutionnel, comme FitzGerald, d'un unioniste libéral il existe donc entre ces courants un accord sur l'influence que la Communauté européenne a sur la question irlandaise, et plus précisément une conviction selon laquelle l'adhésion à la Communauté rapprocherait les deux parties de l'Irlande. Cette attitude reflète la théorie très répandue en Europe qui place l'interdépendance économique à la source de l'union politique. Par contre, comme l'élargissement de la Communauté n'a pas fait progresser l'intégration, on a pu constater un recul sur le plan irlandais de la croyance dans les conséquences bénéfiques de la coopération économique européenne entre les deux Irlandes. Malgré cela, FitzGerald continue de croire que la dimension européenne aura des effets sur la situation constitutionnelle de l'Irlande du Nord. Lors d'une conférence prononcée à la mémoire de John Whyte, en 1990, FitzGerald revenait sur la thèse selon laquelle l'adhésion à la Communauté européenne exigerait, dans l'intérêt de l'Irlande du Nord, un réexamen de ses relations politiques traditionnelles: "Dans l'intérêt de l'Irlande du Nord, disait-il, il est essentiel que celle-ci ait un accès direct à la prise de décisions au sein de la Communauté. II ne me semble pas que cette exigence soit satisfaite par la présente structure qui exige que les intérêts de l'Irlande du Nord soient exclusivement représentés par le gouvernement britannique. Cette exigence ne serait pas non plus satisfaite par la création d'un État nord-irlandais indépendant, car les deux communautés de l'Irlande du Nord et les deux Etats concernés apprécient suffisamment les risques et dangers d'une telle solution pour la rendre inapplicable. Une démarche possible consisterait à mandater l'État irlandais pour représenter, à titre égal, les intérêts des deux Irlande. Ceci exigerait qu'on dépasse les facteurs émotionnels, comme le gouvernement de l'État d'Irlande l'a fait dans sa politique européenne depuis 1973"7.

$\mathrm{Au}$ début, les nationalistes du Nord affichaient, à propos de l'influence potentielle qu'aurait la Communauté européenne sur la situation en Irlande du Nord, une attitude bien plus sceptique que celle de FitzGerald. D'ailleurs, la reprise des "ennuis irlandais" (Irish troubles), en octobre 1968, imposait la recherche de nouvelles solutions politiques pour faire face à la crise en Irlande du Nord. Dans ce contexte, les conséquences à long terme d'une coopération économique via la Communauté européenne paraissaient bien lointaines. L'explosion de violence en Irlande du Nord qui suivit l'introduction de l'internement civil en août 1971, puis l'application de l'administration directe en mars 1972, éclipsèrent sur place l'adhésion du Royaume-Uni et de l'Irlande du Nord, et celle de la république d'Irlande, à la Communauté européenne le 1er janvier 1973. Au même moment et très pertinemment, le gouvernement britannique, après avoir fait référence à l'adhésion prochaine à la Communauté des deux pays, raisonnait dans un cadre où "tout nouvel arrangement pour l'Irlande du Nord devrait, tout en satisfaisant les désirs de l'Irlande du Nord et de la Grande-Bretagne, être acceptable et accepté, dans la mesure du possible, par la république d'Irlande" ${ }^{18}$. Malgré cette dimension européenne évidente de la politique 
britannique en Irlande du Nord, les parties au débat attachèrent peu d'importance à cet aspect des choses.

Cette attitude ne manqua pas d'apparaître lors du référendum de 1975 sur la renégociation par le gouvernement travailliste britannique des termes de l'adhésion. En effet, la population de l'Irlande du Nord, partie constituante du Royaume-Uni, participait au vote, et le gouvernement avait décidé de comptabiliser le vote région par région, ce qui permettait d'isoler le vote de l'Irlande du Nord. Mais la question de savoir si le Royaume-Uni devait rester membre de la Communauté ne recouvrait pas les clivages partisans de la province. Il y avait au contraire une corrélation entre le soutien à l'adhésion à la Communauté d'une part et, d'autre part, le désir d'un partage du pouvoir qui ne tienne pas compte des divisions partisanes et permette de débloquer la situation politique en Irlande du Nord. Ainsi, soutenant l'adhésion, on trouvait ce qui subsistait de l'opinion unioniste libérale, l'Alliance Party et le SDLP. Par contre, la plupart de ceux qui rejetaient tout partage du pouvoir, et notamment le chef du Democratic Unionist Party (DUP), Ian Paisley, s'y opposaient. Certes, il y avait quelques exceptions notables dans cette répartition. Deux des membres influents du SDLP, Gerry Fitt et Paddy Devlin, étaient contre l'adhésion du Royaume-Uni sous prétexte que celleci constituerait un obstacle à la mise en oeuvre de la politique socialiste en matière économique qu'ils souhaitaient avec une partie significative de la gauche britannique. Leur attitude exprimait l'espoir caressé par une fraction du SDLP selon lequel un gouvernement britannique d'inspiration socialiste finirait par dépasser les divisions sectaires de l'Irlande du Nord et faciliterait la solution du conflit. Même si cette opposition n'avait pas existé, l'engagement limité des partis dans la campagne pour le référendum laissait prévoir en Irlande du Nord la victoire des "non". La surprise fut donc grande lorsqu'une légère majorité du "oui" se dégagea. Par contre, la participation peu élevée de l'électorat était le reflet du faible intérêt qu'avait provoqué la campagne référendaire. En fait, la participation était une des moins élevées de toutes les régions du Royaume-Uni. Bien entendu, même si le résultat du vote de l'Irlande du Nord avait été inversé, la cause était entendue. Cependant, si l'Irlande du Nord avait voté "non", se différenciant ainsi de l'Angleterre, de l'Écosse et du pays de Galles, aurait-il été légitime d'en conclure que l'Irlande du Nord n'était pas politiquement en pleine harmonie avec le reste du Royaume-Uni?

En 1976, les résultats d'un sondage en Irlande du Nord montraient que l'attitude envers la Communauté européenne ne recoupait pas la coupure partisane. Ceci dit les catholiques étaient un peu moins défavorables à la Communauté que les protestants ${ }^{9}$ : en effet, $26,7 \%$ des catholiques contre seulement $16,4 \%$ des protestants considéraient que l'appartenance à la Communauté favoriserait la solution des problèmes de l'Irlande du Nord ${ }^{10}$. Le scepticisme restait dominant, lorsqu'en 1979, la république d'Irlande adhéra au « serpent 》 monétaire européen, tandis que la Grande-Bretagne se maintenait à l'écart. En effet, la livre sterling et la livre irlandaise n'étant plus liées, cette situation nouvelle grossissait d'une dimension économique la séparation des deux pays. Dans ce contexte, une convergence politique entre Nord et Sud par le biais de la Communauté paraissait très aléatoire. Une enquête effectuée en 1982 concluait que "vu l'incapacité de la CEE à promouvoir, jusqu'à ce jour, une réelle intégration politique parmi ses propres membres, il paraît illusoire de s'attendre à une contribution sérieuse dans la recherche d'une solution politique pour l'Irlande du Nord"11. Pourtant, la dimension européenne avait acquis entre-temps une signification inattendue en Irlande du Nord par le truchement des élections directes pour le Parlement européen. 
La première de ces élections eut lieu en juin 1979. A l'inverse de ce qui s'était passé lors du référendum, l'Irlande du Nord participa plus largement à ce vote que le reste du Royaume-Uni. En fait, il ne s'agissait pas d'un regain d'intérêt pour les affaires de l'Europe, mais d'un épisode de la lutte politique en Irlande du Nord. Unique circonscription électorale, l'Irlande du Nord était appelée à élire à la représentation proportionnelle, trois membres du Parlement européen. Lan Paisley du DUP arrivait en tête avec $30 \%$ des suffrages, John Hume du SDLP n'était pas loin derrière avec $24,6 \%$, c'est-à-dire un score plus important que celui obtenu par le SDLP en mai lors des élections au Parlement britannique (Westminster). John Taylor, de l'Ulster Unionist Party, était le troisième candidat élu. A l'époque, John Hume n'était que le chef-adjoint du SDLP. Le succès de Hume aux élections européennes "confirmait qu'il était reconnu par le peuple comme le vrai chef de l'opinion nationaliste du Nord"12. Fitt démissionna du SDLP un peu plus tard dans l'année, pour protester contre l'attitude négative du parti au sujet d'une nouvelle initiative politique britannique qui n'était pas assortie d'une mention de la spécificité irlandaise. C'est alors que Hume devint chef du parti.

Le nouveau Parlement européen, élu au suffrage direct, était fondamentalement différent de son prédécesseur, bien qu'il en ait conservé l'étroitesse des compétences. Pendant une brève période, en 1973-4, Rafton Pounder, un unioniste libéral, avait été le seul ressortissant de l'Irlande du Nord à siéger à l'Assemblée européenne. II le faisait d'ailleurs en tant que membre de la délégation du parti conservateur britannique. De toute façon, cette Assemblée ne s'était pas immiscée sur un terrain politique qui ne correspondait pas à la ligne de conduite habituelle de la Communauté. Le Parlement européen, élu en 1979, n'aura pas ces scrupules. En mars 1980, un membre irlandais du parlement, Neil Blaney, tentait vainement d'ouvrir un débat sur l'Irlande du Nord. Cependant, le Parlement changeait d'avis lors de la crise provoquée par une grève de la faim de prisonniers de l'IRA en 1981. En 1982, le Parlement adoptait une résolution interdisant l'utilisation de balles plastiques. En février 1983 il allait même plus loin. Le Parlement décidait qu'une enquête serait entreprise par sa Commission des affaires politiques sur les problèmes politiques et économiques de I'Irlande du Nord. Cette décision indisposait le gouvernement britannique, qui dénonçait l'enquête comme une ingérence dans les affaires internes du Royaume-Uni. Néanmoins, l'enquête eut lieu, avec Niels Haagerup comme rapporteur. Son projet de rapport fut publié en décembre 1983 .

Lorsque l'Europe intervient

Le rapport Haagerup est un document rigoureusement équilibré, tant dans son analyse du conflit que dans les prudentes propositions qu'il fait. Néanmoins, certains aspects du rapport furent durement ressentis par les unionistes; non seulement l'Irlande du Nord était qualifiée de "bizarrerie constitutionnelle"13,mais le rapport recommandait "d'établir, politiquement, juridiquement ou autrement, des co-responsabilités angloirlandaises dans des domaines spécifiques"14. Le rapport fut discuté par le Parlement européen en mars 1984. Une résolution en adoptant les constatations et les recommandations fut votée par 124 voix contre 3, et 63 abstentions. Cet appui massif fut assez généralement considéré comme un triomphe pour le chef du SDLP, John Hume, qui, de surcroît, était un membre influent et très en vue du groupe socialiste au sein du Parlement. Ce climat, très apprécié par le SDLP et le gouvernement irlandais, augmentait la pression internationale sur la Grande-Bretagne pour l'amener à conclure un accord reconnaissant l'intérêt légitime de la république dans les affaires de l'Irlande du Nord. 
Les succès de Hume en tant que député européen l'ont aidé à contrecarrer le chef du Sinn Fein lors des secondes élections directes au Parlement européen. Hume obtenait $22,1 \%$ du vote contre 13,3 \% pour le candidat du Sinn Fein, Danny Morrison, dont le but avoué avait été de le battre. C'était la première défaite électorale importante du Sinn Fein depuis qu'il avait entrepris de contester les élections de 1982 pour l'Assemblée de l'Irlande du Nord. La situation de Hume était encore renforcée par I'accord angloirlandais de 1985 accueilli avec enthousiasme par le Parlement européen. En 1989, aux élections pour le Parlement européen, Hume obtenait 25,5\% des voix contre 9,1 pour Morrison. Un mois auparavant, aux élections locales, les candidats du SDLP avaient recueilli $21 \%$ des votes contre $11,2 \%$ pour les candidats du Sinn Fein ${ }^{15}$. La différence entre les deux résultats s'explique par la capacité du SDLP à élargir sa base électorale grâce à son action au niveau européen. En obtenant des fonds européens pour l'Irlande du Nord, Hume renforçait politiquement la position du nationalisme constitutionnel. En fait, au cours des années 80, il devint évident que le Parlement européen constituait un forum idéal pour faire avancer les intérêts du nationalisme constitutionnel. Il y avait concordance entre l'idéal d'une Irlande unifiée par des moyens pacifiques et celui, plus large, de l'élimination des frontières et des divisions en Europe. Deux universitaires opposés au nationalisme s'exprimaient ainsi : "des politiciens dans le camp nationaliste ont bien réussi, en utilisant un langage et symbolisme "européaniste", à vendre un produit qui est fondamentalement de teneur traditionnelle"16. Les vues du SDLP coïncidaient avec la sensibilité des députés européens aux violations des droits de l'homme par l'État et leur hostilité à l'IRA comme organisation terroriste. In fine, la conviction du SDLP selon laquelle des changements constitutionnels étaient possibles en employant des moyens pacifiques était vérifiée par le processus européen d'intégration. La ratification en 1987 de l'Acte unique européen de 1985 renforçait la spéculation sur la forme future de la Communauté européenne. Il était généralement admis que l'introduction d'un marché unique à la fin de 1992 - conséquence de l'Acte unique exigeait, comme contrepoids, le développement de la politique sociale de la Communauté, si on souhaitait que les intérêts des régions pauvres soient protégés. Le Président de la Commission européenne, Jacques Delors, considérait que ce développement imposait une restructuration radicale et un renforcement des institutions de la Communauté. Ses idées étaient à l'opposé de celles du Premier ministre britannique, Margaret Thatcher, qui les considérait comme une menace pour l'État-nation. Dans ce débat, Hume donnait tout son appui à Delors et ce faisait un ardent défenseur de "l'Europe des régions", notion qui avait le vent en poupe. En 1987, Hume présentait un rapport au Parlement européen concernant les problèmes régionaux de l'Irlande. Ce rapport recommandait la création de neuf autorités régionales investies de pouvoirs exécutifs et de planification. Dans sa contribution à un ouvrage collectif sur l'Irlande des années 90 , il considérait que "les tendances des processus économiques exigent une politisation démocratique au niveau macro, et, en même temps, la facilitent et l'exigent au niveau régional et local". Cependant son chapitre ne fait aucune référence à l'impact que de tels développements pourraient avoir sur le problème de l'Irlande du Nord, bien que ce thème soit traité par plusieurs autres contributeurs". Lorsqu'en janvier 1990 le Secrétaire d'État pour I'Irlande du Nord, Peter Brooke, déclencha une initiative politique, il la fondait sur un accord du SDLP et des unionistes selon lequel toute négociation concernant l'avenir de la province serait menée en fonction de trois types de relations: les relations entre les deux communautés de l'Irlande du Nord; les relations entre les deux parties de 
l'Irlande ; les relations entre le Royaume-Uni et la république d'Irlande. L'absence de toute référence à l'Europe comme quatrième type de relations n'était pas entièrement surprenante, étant donné l'hostilité à l'intégration européenne affichée autant par les unionistes que par le gouvernement britannique. Cette lacune était signalée par de nombreux commentateurs, notamment après 1 'échec en 1991 des discussions entre partis et le succès des négociations menant au traité de Maastricht. Richard Kearney qualifiait l'Europe des régions de "dernière et de meilleure chance, dans un avenir prévisible, de résoudre la crise de l'Irlande du Nord" ${ }^{17}$. Le rédacteur de Fortnight, Robin Wilson, partageait cet enthousiasme pour l'Europe comme exutoire des "ennuis" de l'Irlande du Nord: "L'autobus de l'Ulster n'a également qu'une destination. Ce n'est qu'un arrêt de plus au terminal à côté de Munster, Bade-Wurttemberg, Catalogne et beaucoup d'autres. Ce seront peut-être les événements d'Écosse, plutôt que d'ailleurs, qui feront démarrer le moteurS Toute la question est de savoir à quelle vitesse - et à quel coût en vies estropiées - il arrivera en bout du chemin"18.

Ce point de vue n'était pas partagé par tous. Dans le même numéro de Fortnight, l'ancien chef du bureau de Belfast de la Commission européenne, Dennis Kennedy, critiquait ces idées. I1 faisait remarquer que le comité des régions, créé par le traité de Maastricht, n'était qu'un organisme consultatif et qu'il se trouvait "éloigné d'une distance de plusieurs Maastricht d'une véritable deuxième chambre du Parlement européen ${ }^{19}$. Kennedy soulignait la primauté des États membres qui, en dépit de la rhétorique entourant le traité, était conservée. Il s'employait à démasquer les contes de fée concernant la fin de l'État-nation, cherchant par là autant à dégonfler les espoirs des nationalistes qu'à calmer les craintes des unionistes par rapport à la Communauté européenne.

Bien que la critique de Kennedy soit largement justifiée et bien ciblée, eu égard à certaines illusions entretenues autour du traité de Maastricht, l'impact des notions de régionalisation et décentralisation avancées par Kearney et Wilson restait néanmoins très fort. D'ailleurs, les changements en Europe de l'Est et dans l'ancienne Union soviétique ne faisaient qu'apporter de l'eau au moulin: on pouvait facilement interpréter les événements survenus dans ces régions comme une leçon destinée à instruire le reste du monde des dangers que comporte un nationalisme exacerbé. La dissolution de l'Union soviétique, et surtout celle de la Yougoslavie, brisaient le tabou que la communauté internationale éprouvait à l'égard de toute sécession, et légitimait le droit à l'autodétermination de groupes établis dans des régions ou sous-ensembles bien définis d'un État. Puisque la Croatie et la Slovénie avaient été acceptées comme membres des Nations unies, pourquoi le même droit serait-il refusé à l'Écosse, la Corse, la Catalogne, au Québec, pour ne mentionner que des cas où il existe un soutien d'une partie de la population aux thèses nationalistes régionales? Le cas de l'Écosse avait une signification toute particulière pour l'Irlande du Nord, car une sécession de l'Écosse menacerait l'existence même du Royaume-Uni. Les élections générales britanniques d'avril 1992 qui entraînèrent un renforcement du parti conservateur en Écosse et une progression d'une modestie inattendue du parti national écossais, mirent fin, au moins pour l'instant, à toute possibilité de sécession de l'Écosse, mais il reste toujours en Écosse un courant important en faveur d'une dévolution du pouvoir. Cette éventualité retient l'intérêt des unionistes en Irlande du Nord qui souhaiteraient que la dévolution pour l'Écosse et pour l'Irlande du Nord soit considérée dans le cadre plus large d'un schéma de décentralisation au sein du Royaume-Uni, ce qui soulignerait les liens de l'Irlande du Nord avec le reste du pays ${ }^{20}$. 
L'Europe comme solution ? Après les élections générales britanniques d'avril 1992, les pourparlers inter-partis concernant l'avenir de l'Irlande du Nord reprirent. En 1991, ils avaient échoué sur des points de procédure, sans que le fond du problème ait pu être abordé. Cette fois-ci, les partis purent enfin formuler leurs idées sur la manière dont l'Irlande du Nord devait être gouvernée. Afin de faciliter les discussions, les partis s'étaient mis d'accord sur la confidentialité des propositions qu'ils avanceraient. Cependant, malgré cet accord, des fuites se produisirent et les propositions du SDLP, puis celles des autres partis furent publiées dans la presse. Le SDLP proposait que l'Irlande du Nord soit gouvernée par une commission exécutive, calquée - sans pour autant en être la copie conforme ${ }^{21}$ - sur la Commission européenne. Il proposait que cette commission reflète "les préférences démocratiquement exprimées de l'électorat nord-irlandais", et également qu'elle traite "les relations externes cruciales $^{22}$. II suggérait que la commission soit composée de six membres, dont trois seraient élus de la même manière que les trois députés européens de la province, les trois autres étant nommés respectivement par le gouvernement britannique, le gouvernement irlandais et la Communauté européenne. II proposait également la création d'une assemblée parlementaire calquée sur le parlement européen. Simultanément, le SDLP indiquait qu'il ferait ultérieurement des propositions sur les liens entre les deux parties de l'Irlande. En somme, ses propositions se limitaient pour le moment aux questions concernant le gouvernement intérieur de l'Irlande du Nord. Un article de l'Irish Times de juillet 1992 expliquait que le plan du SDLP était fondé sur des documents préparés en 1990 et 1991 par deux anciens membres d'un groupe de travail à Bruxelles avec les commissaires européens ${ }^{23}$.

Les autres partis politiques de l'Irlande du Nord adoptaient une attitude négative à l'égard du plan du SDLP. Selon certaines sources, le gouvernement britannique était également défavorable à ces propositions ${ }^{24}$. Pour Dennis Kennedy, le modèle européen d'un groupement de souveraineté parmi les douze États membres n'était pas transférable à l'Irlande du Nord dont la société était profondément divisée. Par ailleurs, il considérait que la ressemblance entre le plan du SDLP et les institutions européennes étaient davantage une question de terminologie qu'une similitude réelle; le plan "n'était que de très loin, et peut-être pas du tout, fondé sur le modèle de la Communauté européenne"25. Il faisait remarquer que l'institution cruciale de la Communauté européenne était le Conseil des ministres, et qu'il n'y avait aucun équivalent véritable de cet organisme dans le plan du SDLP. Répliquant à Kennedy, Paul Arthur expliquait que le plan du SDLP devait être considéré comme une déclaration préliminaire à un processus de négociation et que comme tel il constituait "un bond imaginatif et libérateur par rapport à une politique de café du commerce"26. Paul Arthur ne tentait pas de défendre les propositions du SDLP dans leur détail, mais mettait l'accent sur la pertinence de la dimension européenne pour résoudre le problème nord-irlandais. A l'appui de sa thèse, il citait la conférence inaugurale du professeur Elizabeth Meehan à l'université Queen's de Belfast, dans laquelle cette universitaire développait l'idée que la Communauté européenne créait une nouvelle forme de citoyenneté "ni nationale, ni cosmopolite", mais "multiple pour autant qu'elle permettait aux différentes identités qui nous habitent de s'exprimer ${ }^{27}$. En d'autres termes, la Communauté européenne pouvait créer un contexte dans lequel protestants et catholiques d'Irlande du Nord accommoderaient leurs identités nationales sans qu'il $\mathrm{y}$ ait, pour autant, sacrifice des uns ni des autres. 
Tom Hadden et Kevin Boyle, qui dans leurs écrits avaient fortement soutenu les accords anglo-irlandais de $1985^{28}$, défendaient également l'opinion que la dimension européenne était indispensable pour résoudre le conflit de l'Irlande du Nord. Ils considéraient le plan du SDLP comme "un rappel utile sur le fait qu'il fallait prendre en considération sérieusement tous les aspects de la dimension européenne"29. Cependant, ils ne pensaient pas qu'une intervention directe de la Communauté européenne fut nécessaire, ou même souhaitable, pour faire jouer la dimension européenne, car il y a plusieurs voies possibles pour impliquer la Communauté européenne. Ainsi l'argument en faveur d'une décentralisation des pouvoirs au profit des organismes locaux de l'Irlande du Nord était nourri par le fameux principe de subsidiarité adopté par la Communauté - c'est-à-dire la prise de décision au niveau le plus bas, compte tenu de l'efficacité. En même temps, la question du statut de l'Irlande du Nord pouvait utilement être envisagée sous l'angle "d'un statut plus spécifiquement européen en tant que région peuplée d'un mélange des souches britanniques et irlandaises" ${ }^{30}$. Le Conseil de l'Europe, à travers la Convention européenne des droits de l'homme, avait un rôle à jouer en Irlande du Nord. Par ailleurs, Tom Hadden et Kevin Boyle considéraient que la dimension européenne n'était pas limitée à la Communauté. Le but étant la résolution de conflits et le statut des minorités, les mécanismes qui étaient créés pour l'Europe de l'Est par la Conférence sur la sécurité et la coopération en Europe étaient également applicables pour l'Irlande du Nord.

Conclusion

A la base des discussions en Irlande du Nord sur l'influence potentielle de la dimension européenne, il y avait l'hypothèse que les développements en Europe rogneraient la puissance de l'État-nation en tant que module de souveraineté territoriale. Les libéraux tels que Hadden, Boyle, Meehan et Wilson espéraient que ce processus d'érosion aiderait à désamorcer le conflit en Irlande du Nord, en diminuant la signification du statut du territoire, qu'il soit britannique ou qu'il soit irlandais. Les critiques de cette vue libérale s'interrogeaient sur la pertinence concrète de ce processus d'érosion sur le conflit et considéraient que son effet serait d'augmenter le sentiment d'insécurité parmi les unionistes et, par voie de conséquence, d'exacerber, plutôt que de désamorcer, le conflit. Les unionistes soupçonnaient que l'enthousiasme du SDLP pour la dimension européenne cachait l'objectif traditionnel des nationalistes celui d'une Irlande indépendante et unie. Les partisans du Sinn Fein, au contraire, craignaient que l'union européenne ne soit un prétexte pour abandonner l'idéal d'une Irlande indépendante et unie. Malgré ses liens avec nombre de ces mouvements séparatistes qui sont favorables à l'Europe des régions, la méfiance du Sinn Fein à l'égard de cette notion lui donne sur ce point une place à part. II restait fermement attaché à l'interprétation anticolonialiste du conflit en Irlande du Nord, et, par conséquent, attachait plus d'importance aux appels adressés aux Nations unies qu'à tout engagement de la Communauté européenne. Towards a Lasting Peace in Ire1and document de politique générale du Sinn Fein, paru en 1992, ne contenait qu'une brève référence à la dimension européenne. Son idée principale était que les événements en Europe de l'Est soulignaient la signification autant que la puissance du principe de l'autodétermination. Il mit l'accent sur la réunification de l'Allemagne, faisant remarquer que la séparation de l'Irlande était "aussi irrégulière en jurisprudence internationale"131.

La principale ligne de clivage de l'opinion semble se situer entre, d'une part, ceux qui considèrent que la dimension européenne a changé la nature du débat sur l'avenir de 
I'Irlande du Nord et d'autre part, ceux qui pensent que l'Europe n'a pas de pertinence pour le conflit et qu'elle sert les objectifs nationalistes. John Hume, dans un article qui soutient Maastricht avant le référendum dans la république, évoque avec éloquence pour défendre sa position : "Les notions de souveraineté et d'indépendance, qui sont au c ?ur des guerres européennes et de la querelle anglo-irlandaise, ont changé de signification. I1 existe actuellement une souveraineté partagée et une interdépendance, et nous cheminons inévitablement vers l'établissement des États unis d'Europe; pour nous en Irlande, cela signifie que nous devons nous débarrasser de nos obsessions à l'encontre de la Grande-Bretagne et reconstruire nos liens avec le reste de l'Europe. Notre identité nous a forgé une communauté d'intérêts économiques et culturels avec maintes régions européennes, ce qui nous prédestine à la phase suivante de cette évolution - la régionalisation de l'Europe ${ }^{32}$.

Pour les unionistes, ces paroles de Hume comportent un sens nationaliste sous jacent, car tout affaiblissement des liens avec la Grande-Bretagne leur parait être un pas pour l'union avec l'Irlande. Cette méfiance semble difficile à négliger aussi longtemps que le SDLP maintient son objectif d'une Irlande unie. La méfiance des unionistes envers l'Europe justifie, par elle-même, du moins partiellement, le point de vue que l'intégration ne change pas (et ne changera pas dans un avenir prévisible) la nature du conflit en Irlande du Nord. Cependant, on oublie un aspect crucial du jeu de l'Europe son impact sur la république d'Irlande.

En Irlande du Nord, l'adhésion à la Communauté européenne en transformant le statut de la République dans le monde, a modifié les mentalités. Garrett FitzGerald, dans son autobiographie de 1991, fait remarquer que l'indépendance politique n'a pas rendu la République indépendante de la Grande-Bretagne en matière d'économie, mais que cet objectif" a été atteint par son adhésion à la Communauté, au sein de laquelle nous sommes maintenant à pied d'égalité avec notre voisin" ${ }^{13}$. Cette amélioration du statut de la République, à la suite de son adhésion à la Communauté, constituait un facteur significatif dans l'évolution de son attitude envers l'Irlande du Nord ; la scission du pays en deux était ressentie moins péniblement; elle cessait d'être perçue comme le symbole de la sujétion colonialiste de l'Irlande à l'Angleterre, - cette analogie ne s'appliquant pas à un membre du club privilégié des riches nations européennes. Simultanément, l'opinion du Sud se détachait du climat de violence dominant le Nord. Après l'accord anglo-irlandais de 1985 accord qui admettait un droit de regard de la République dans la détermination des politiques suivies par la Grande-Bretagne en Irlande du Nord, le Sinn Fein se marginalisait au Sud. De plus en plus fréquemment on voit le drapeau de la Communauté à côté du drapeau tricolore de la République, sinon à sa place. Plus les Irlandais se voient en "bons Européens", plus l'opinion du Sud rejette les nationalismes de tout genre. Par voie de conséquence, on peut assister au spectacle dérisoire de politiciens irlandais dénonçant le nationalisme britannique dans le contexte des débats sur l'avenir de l'Europe ${ }^{34}$.

Pour la majorité des catholiques du Nord, ce changement d'attitude du Sud apporte du bon et du moins bon. D'une part, les attitudes plus accommodantes du Sud envers les unionistes éliminent un des obstacles à un accord sur les institutions politiques en Irlande du Nord, par dessus les divisions sectaires. Actuellement, il n'existe pas grand risque qu'un accord du SDLP avec les unionistes en Irlande du Nord soit dénoncé par une partie significative de l'opinion du Sud. Par contre, le SDLP court le risque d'être débordé par le désir du gouvernement irlandais d'arriver à un arrangement avec les unionistes. Sans le soutien du Sud, le SDLP pourrait se trouver isolé dans ses 
négociations concernant l'avenir de l'Irlande du Nord. En fait, des rumeurs circulent indiquant que cette éventualité se produit au détriment du modèle européen proposé par le SDLP pour le gouvernement futur de l'Irlande du Nord ${ }^{35}$. Il serait ironique de constater que la prise en compte de la dimension européenne qui traduit un changement dans l'attitude du Sud a comme conséquence de gêner les nationalistes du Nord dans leur recherche d'une solution européenne au problème irlandais. Cependant, l'impact de la dimension européenne comporte tant de variables interdépendantes qu'il serait absurde de vouloir prédire son influence sur le déroulement futur du conflit de l'Irlande du Nord. En fin de compte, deux choses sont certaines : d'une part, la majorité des catholiques de l'Irlande du Nord perçoit à présent que l'Europe a un rôle significatif à jouer dans la solution du conflit; d'autre part, les progrès de l'intégration européenne ont avantagé le SDLP - pro-européen - et ont consolidé son influence dans la communauté catholique.

\section{NOTES}

1. I. Brian Walker, "Historic Changes as North and South Differ on European Union, Dublin", The Irish Times, 29juin 1992.

2. An Phoblacht, Dublin, 11 juin 1992

3. Ibid.

4. Northern Ireland House of Commons Debates, Vol. 83, Col. 990, 8 décembre 1971.

5. Garret FitzGerald, Towards a New Ireland, London, Charles Knight, 1972, p. 104.

6. Ibid., pp. 111-112.

7. Garret FitzGerald, What Makes Polilics Tick ? ; Interests, Ideals, or Emotions ? Belfast, Queen's Politics Occasional Paper nº3 ; 1991, pp. 26-7.

8. The Future of Northern Ireland, a Paper for Discussion, Londres, HM50, 1972, p. 34.

9. Edward Moxon-Browne,Nation, Class And Creed in Northern Ireland, Aldershot, Gower, 1983, p. 158.

10. Ibid., p. 161

11. Northern Ireland: Problems and Perspectives, Londres, Institute for the Study of Conflict, 1982, p. 42.

12. Barry White, John Hume : Statesman of the Troubles, Belfast, Blackstaff Press, 1984, p. 204.

13. Report drawn up on behalf (the Political Affairs Commitee on the situation in Northern Ireland (Rapporteur : N.J. Haagerup), European Parliament Working Documents 1983-1984, Document 1-1526/83 (19 mars 1984), p. 37.

14. Ibid., p. 73.

15. Tiré de Michael Marsh et Rick Wilford, "Irish Political Data, 1989", Irish Political Sludies, Vol. 5, 1990, pp. 140-3.

16. Paul Bew et Henry Panerson, "Ireland in the 1990's - North and South" dans Richard hearney (ed.), Across the Frontiers : Ireland in the 1990s, Dublin, Wolfhound Press, 1988, pp. 89-90.

17. Cité dans Fortnight (Belfast) n³02,janvier 1992, p. 5. 
18. Robin Wilson, "This Bus Can Not Wait", Fortnight, janvier 1992, p. 5.

19. Dennis Kennedy, "Forget Fairy Tales", Fortnight, janvier 1992, p. 10.

20. Voir, par exemple, Derek Bateman, "Unionists Forge United Front with Scots", Londres, The Observer, 24 mai 1992.23. "

21. SDLP Plan for North Government", The Irish Times, 13 mai 1992.

22. Ibid.

23. Joe Carroll, "EC Offcials Worked on SDLP Model", The Irish Times, 10 juillet 1992.

24. Voir, par exemple, Mary Holland, "Threadbare Truce for Ulster Talks Hangs by a Strand", The Observer, 5 juillet 1992.

25. Dennis Kennedy, "Why the European Model Has Little Relevance for North", The Irish Times, 25 mai 1992.

26. Paul Arthur, "Setting NI Scene for a New Way of Thinking, The Irish Times, 28 mai 1992.

27. Ibid.

28. Voir, par exemple, Kevin Boyle et Tom Hadden, Ireland : a Positive Proposal, Penguin, Harmondsworth,1985.

29. Tom Hadden et Kevin Boyle, "Fourth Dimension, Fortnight, n 307,juin 1992, p. 25.

30. Ibid.

31. Towards a Lasling Peace in Ireland. Dublin, Sinn Fein, 1992, p. 14

32. John Hume, "Irish Problem is European in its Origins", The Irish Times, 8juin 1992.

33. Garrett FitzGerald, All in a Life, Dublin, Gill and MacMillan, 1991, p. 176.

34. Voir, par exemple, Peter Sutherland, "Stronger than Nations", Londres, Financial Times, 28 mai 1992.

35. Voir, par exemple, Ed Maloney, "Unionists Perceive Dublin-SDLP Rift", Dublin the Sunday Tribune, 12 juillet 1992.

\section{RÉSUMÉS}

L'accord anglo-irlandais de 1985 a aidé à restreindre le conflit politique en Irlande du Nord, par contre l'accord n'a pas solutionné la question du statut politique de l'Irlande du Nord ni l'entente entre les partis politiques sur la question de la redistribution des pouvoirs politiques. De ce fait, il y a un intérêt marqué dans la possibilité que l'union européenne peut fournir un cadre équitable à une solution plus complète à la question irlandaise. La minorité catholique est divisée autour de la question du rôle de l'Europe. Sinn Fein considère que l'intégration européenne représente un obstacle à son but d'une Irlande unifiée. Par contre, le parti social démocrate travailliste appuie la vision d'une Europe conçue en termes de régions comme modèle d'accommodement politique entre protestants et catholiques de l'Irlande du Nord. L'enthousiasme pour l'intégration politique témoigné par le parti social démocrate travailliste est fondé sur les avantages politiques qu'il retire de l'avancement de l'intégration européenne.

The Anglo-lrish Agreement of 1985 has helped containing the Northern Ireland conflict, but it has neither resolved the issue of Northern Ireland's status as a political entity nor led to an agreement on the redistribution of political power among the parties in Northern Ireland. As a result there has been an increasing interest for the possibility of the European Union providing a 
suitable framework for a more complete solution to the Irish Question. The Catholic minority is divided on the question of Europe's role. Sinn Fein regards the European integration as an obstacle to the achievement of its goal of a united Ireland. By contrast, the Social Democratic Labour Party has enthusiastically supported the notion of a Europe of Regions capable of providing the context for political accommodation between Protestants and Catholics in Northern Ireland. The party's enthusiasm for the European Union is based on the political advantages it has derived from the progress of European integration.

\section{INDEX}

Mots-clés : identité, Nationalismes, religion

Index géographique : Europe, Irlande du Nord, Royaume Uni

Thèmes : Sinn Fein

\section{AUTEUR}

\section{ADRIAN GUELKE}

Maître de conférence à la faculté de science politique de Queen's University à Belfast. 